\title{
Reconstruction of Exposed Frontal Bone Following Mohs Micrographic Surgery
}

\author{
Mario J Sequeira ${ }^{1,2 *}$, and Evan M Sequeira ${ }^{2}$ \\ ${ }^{1}$ Dr. Phillip Frost Department of Dermatology and Cutaneous Surgery, University of Miami, Miller School of Medicine, Miami, FL, USA \\ ${ }^{2}$ Brevard Skin and Cancer Center, Rockledge, FL, USA
}

*Corresponding author: Sequeira MJ, Brevard Skin and Cancer Center, Rockledge, FL, USA, Tel: 321 636 7780; E-mail: masequeira@cfl.rr.com

Received: 18 Nov, 2019 | Accepted: 02 Dec, 2019 | Published: 09 Dec, 2019

Citation: Sequeira MJ, Sequeira EM (2019) Reconstruction of Exposed Frontal Bone Following Mohs Micrographic Surgery. J Clin Cosmet Dermatol 3(3): dx.doi.org/10.16966/2576-2826.143

Copyright: (C) 2019 Sequeira MJ, et al. This is an open-access article distributed under the terms of the Creative Commons Attribution License, which permits unrestricted use, distribution, and reproduction in any medium, provided the original author and source are credited.

\begin{abstract}
Mohs Micrographic Surgery for malignant neoplasms of the head on occasion can result in defects with exposed skull bone. Full thickness wounds devoid of periosteum can be managed by secondary intention healing facilitated by bone chiseling to stimulate granulation tissue, however, the process of full re-epithelialization is lengthy. Elderly patients often are unable to manage such wounds and immediate alternative post surgical reconstructive options must be entertained. We report the use of the bilobed flap as originally described by Esser with planned delayed staged suturing to provide coverage of an exposed frontal bone defect while improving flap survival.
\end{abstract}

Keywords: Frontal bone; Mohs Micrographic Surgery; Bilobed flap; Delayed staged suturing

\section{Introduction}

Although there is a risk of osteomyelitis with delayed healing of exposed forehead bone, such events are rare [1]. There is evidence that secondary intention healing can be safely accomplished on large defects with exposed bone. A retrospective study of 91 such cases with exposed bone of the face and scalp resulted in a successful healing rate of $95 \%$ [2]. Complications included infections (2.7\%) and poor wound healing $(2.7 \%)$, but no cases of osteomyelitis were reported [2]. Healing in clinical situations with exposed bone is accelerated by chiseling the outer table of the bone until pin point bleeding is observed [3]. This is accomplished using a narrow chisel with sharp taps using a mallet [2]. The bone drill and rongeur can also be used $[4,5]$. Bleeding promotes formation of a granulating tissue bed and decreases the distance cells must migrate from the peripheral wound edges to provide epidermal coverage [3].

A direct measurement of eight patients with scalp defects who underwent bone chiseling documented a rough estimate of healing time based on exposed bone width of a factor of 2 times its width in weeks for granulation coverage of bone and a factor of 3 times its width in weeks for full re-epithelialization of the wound [2]. Based on this formula, a defect of $4 \mathrm{~cm}$ could potentially take 8 weeks to develop a granulating bed and 12 weeks to fully reepithelialize. Wound care involves irrigating the wound with tap water or saline, cleansing with soap and water, patting the wound dry followed by application of antibiotic ointment or petrolatum and placement of a non adherent dressing which is changed every second or third day [2]. Hydrogen peroxide is best avoided as it can desiccate the bone [2]. This meticulous wound care is sometimes unachievable by elderly patients necessitating an alternative post surgical repair option.

\section{Case Report}

An 87 year old woman with skin type I/II, history of extensive sun exposure and multiple non melanoma skin cancers was referred to the author's cancer center post excision of an invasive squamous cell carcinoma of the mid superior forehead requiring two Mohs stages to achieve a tumor free plain. Her surgical course was complicated by wound dehiscence with a resulting defect that measured $3.2 \times 2.0$ $\mathrm{cm}$ with exposed bone devoid of periosteum (Figure 1). The patient lived alone and lacked nursing care at home. Repair options were scarce based on her skin atrophy, prior sloughed wound edges due to tension, and limited tissue mobility. A bilobed flap was designed with the axis of the first lobe 90 degrees from the wound and the second lobe on a line 180 degrees with that of the defect allowing recruitment of the only available lax skin (Figure 2).

A regional block was achieved by injecting $1-2 \mathrm{ml}$ of $1 \%$ lidocaine $17 \mathrm{~mm}$ and $27 \mathrm{~mm}$ from the glabellar midline bilaterally just above the superior orbital rim exterior to the bony orbital margin corresponding to the location of the exiting supratrochlear and supraorbital nerves, respectively (Figure 1). The first lobe of the bilobed flap was drawn perpendicular and of equal width as the width of exposed bone. The second lobe was drawn 90 degrees from the first lobe and also of the same width (Figure 2). The flap was designed whereas the second lobe recruited the only area of tissue laxity and the resulting tertiary defect could be closed with minor tension. After local anesthetic infiltration, the incision was carried down to the deep subcutaneous layer along the drawn flap outline. The first lobe was rotated approximately 60 degrees onto the defect providing coverage to the exposed frontal bone with minimal dog ear formation (Figure 3 ). The second lobe was transposed 90 degrees onto the secondary defect. The resulting 


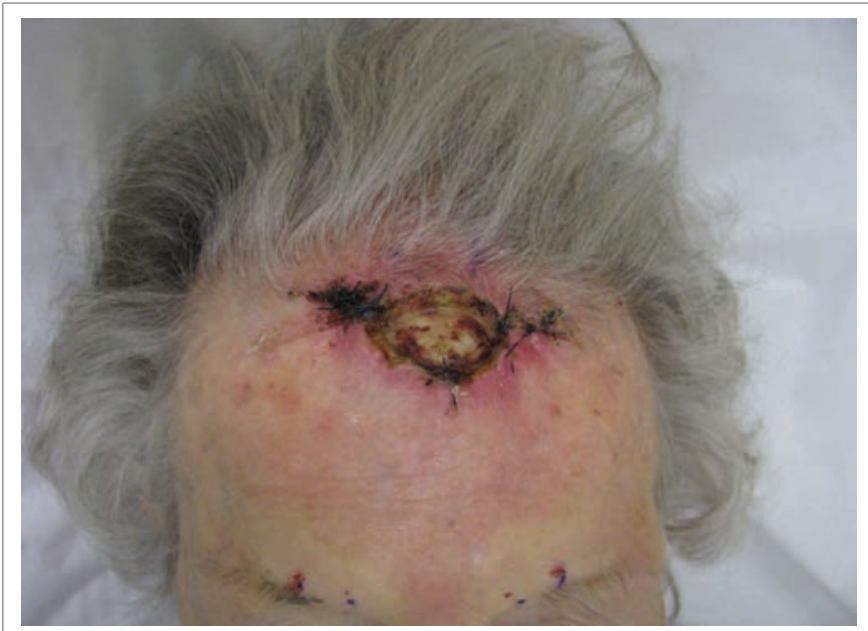

Figure 1: Original presentation with exposed frontal bone.

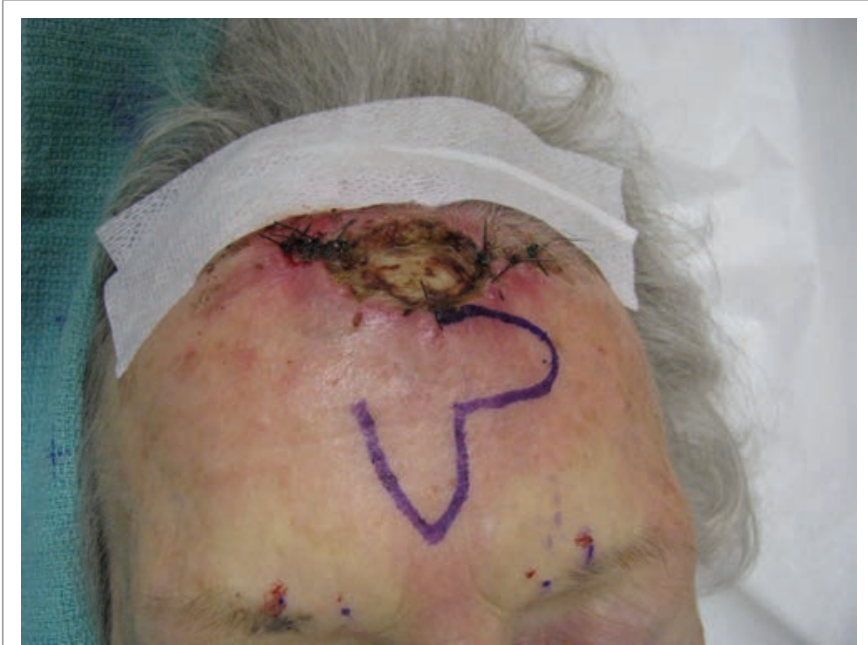

Figure 2: Design of bilobed flap.

tertiary defect was approximated side to side with subcutaneous 4.0 polyglactin 910 sutures. The remaining wound was closed externally with interrupted 4.0 nylon sutures with the exception of two small areas where suturing created blanching on the flap potentially compromising its vasculature (Figure 3 ). A decision was made to allow these small areas to heal by second intent to increase the probability of flap survival (Figure 4). At nine days post operatively the original sutures were removed and the tissue edges of one of the widened areas left to heal by second intent was gently curetted and sutured with three interrupted 4.0 nylon sutures to improve cosmesis without vascular compromise of the now healing flap (Figure 5). These sutures were finally removed one week later. At six month follow up the surgical outcome was excellent (Figure 6).

\section{Discussion}

Repair options for this patient were carefully considered [6]. Unfortunately, a primary intermediate or complex linear closure was not an option due to her history of wound dehiscence and prior wound edge necrosis due to high tension. Different flaps were also entertained (advancement, rotation and transposition) and once again the only area available to recruit for skin closure was inferior and away from the wound edges. It was felt that any movement other than a double

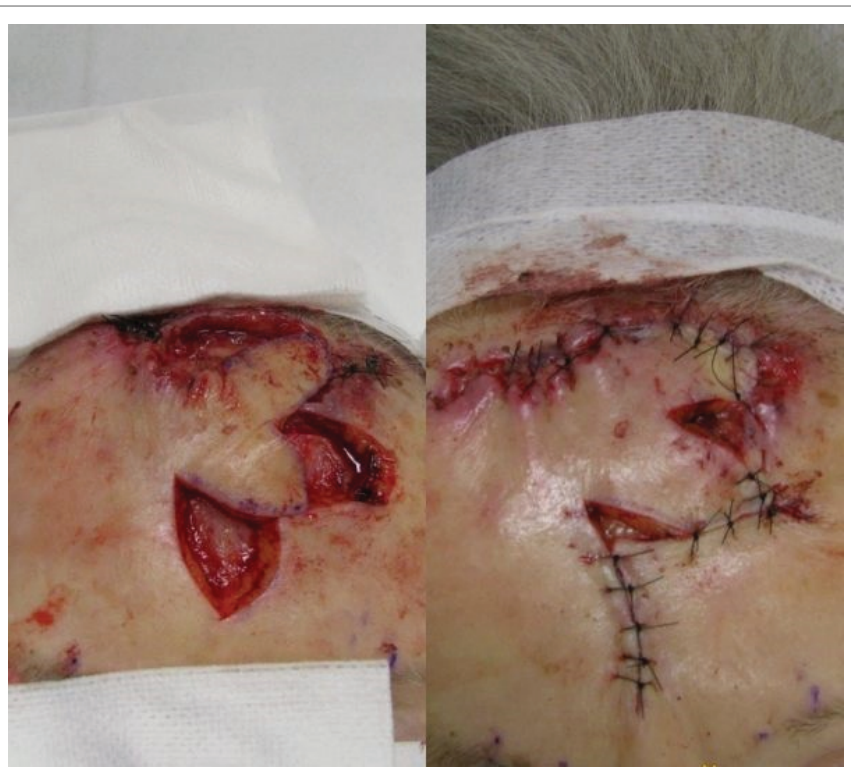

Figure 3: Movement of bilobed flap.

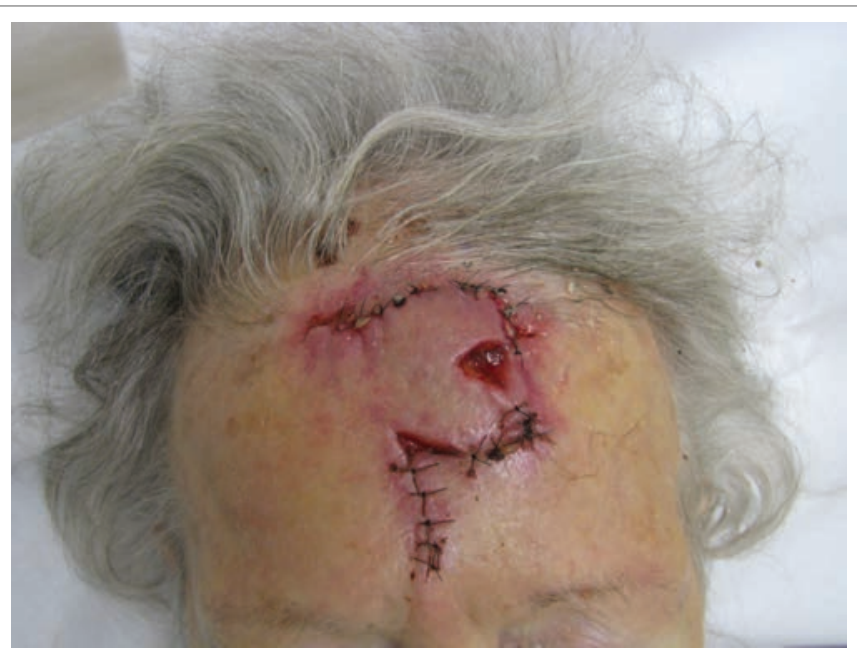

Figure 4: Site left to heal by second intention.

transposition flap (Bilobed) or a large bilateral rotation flap along the hairline would cause high skin tension along the sutured repair. Due to the lack of periosteum and poor vasculature of the exposed frontal bone, a skin graft would not have survived. Burring or chiseling the outer table of the exposed bone to stimulate granulation tissue formation or use of a dermal substitute followed by placement of a split thickness graft at a later time was a viable option but declined by the patient [7]. The use of a bilobed flap with a delayed staged suturing approach was felt to be the best option for an immediate repair.

In the bilobed flap design as described by Esser each lobe is drawn 90 degrees from each other and the second lobe is drawn at an axis of 180 degrees with that of the primary defect. This allows recruitment of lax tissue further away from the defect [8]. The Zitelli design modification of the bilobed flap creates a pivot point and each lobe is drawn 45-50 degrees from each other allowing for a smaller arc of rotation (90-100 degrees) which minimizes dog ear formation and works best on the distal nose [9]. There is flexibility in which design to choose dictated by body region, desired placement of scars, and planned recruitment 


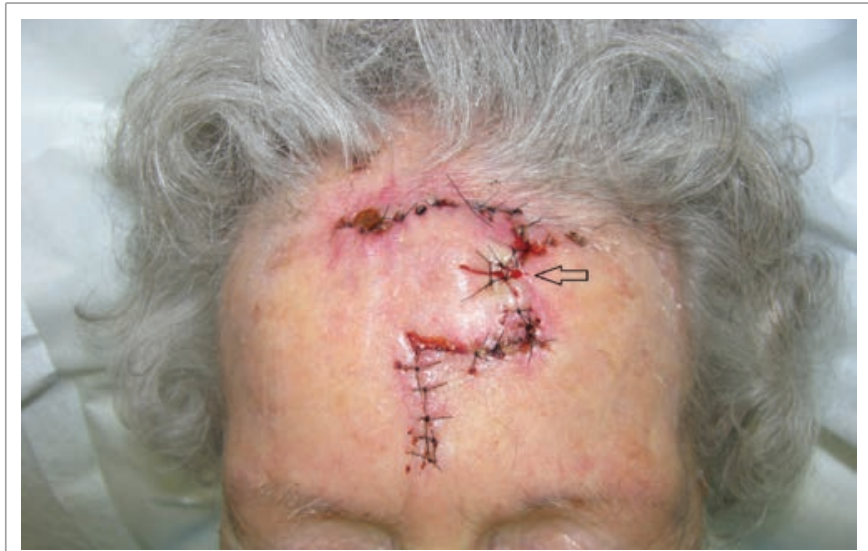

Figure 5: Delayed staged suturing of second intention site.

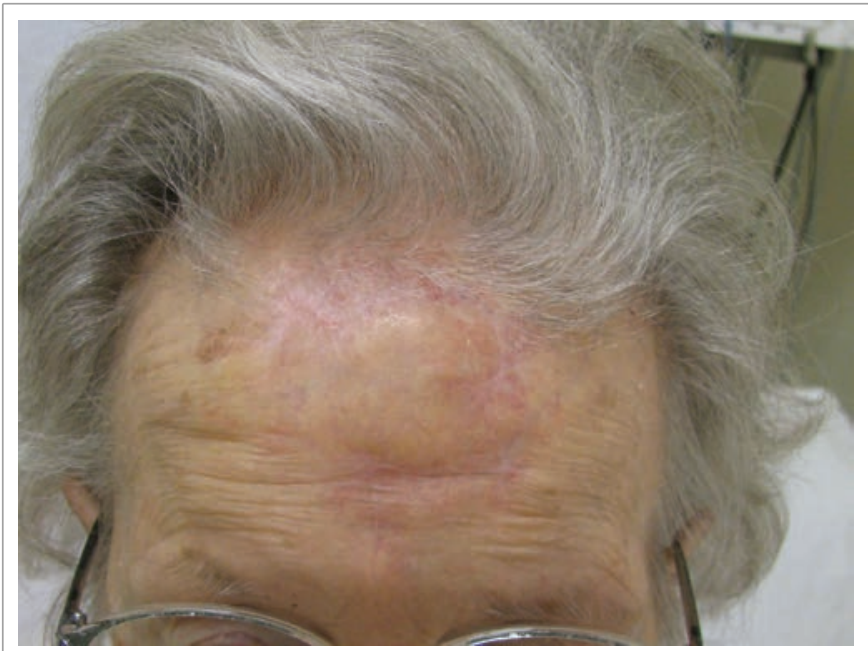

Figure 6: Outcome six months post operatively.

of lax skin. As our case illustrates, flaps do not always have to be fully repaired or sutured under high tension if such compromises vascular perfusion resulting in tissue slough or flap necrosis. Small areas can be left to heal by secondary intention if such maneuver reduces wound tension and improves flap perfusion. A delayed staged suturing of these areas can improve cosmetic outcome by creating narrower scars and can be performed once flap survival is more readily apparent. In our case this was accomplished at 9 days post operatively.

\section{Conclusion}

Our case report highlights the efficient but seldom used forehead bilobed flap with delayed staged suturing to improve flap survival while providing coverage of mid to large defects of the central superior forehead with exposed frontal bone $[10,11]$. Staged suturing decreases the uncertainty of flap survival especially when incorporated in bilobed flap repairs in areas of high skin tension [12].

\section{References}

1. Snyder PA, Alper JC, Albom MJ (1984) Osteomyelitis complicating mohs' chemosurgery. J Am Acad Dermatol 11: 513-516.

2. Snow SN, Stiff MA, Bullen R, Mohs FE, Chao WH (1994) Secondintention healing of exposed facial-scalp bone after mohs surgery for skin cancer: review of ninety-one cases. J Am Acad Dermatol 31: 450-454.

3. Vanderveen EE, Stoner JG, Swanson NA (1983) Chiseling of exposed bone to stimulate granulation tissue after mohs surgery. J Dermatol Surg Oncol 9: 925-928.

4. Latenser J, Snow SN, Mohs FE, Weltman R, Hruza G (1991) Power drills to fenestrate exposed bone to stimulate wound healing. J Dermatol Surg Oncol 17: 265-270.

5. Ceilley RI, Bumsted RM, Panje WM (1983) Delayed skin grafting. J Dermatol Surg Oncol 9: 288-293.

6. Monk EC (2019) Repair of large forehead defect. Dermatol Surg 45: 1097-1099.

7. Monary $\mathrm{P}$, Moro R, Marocolo D, Manganoni AM, Calzavara-Pinton P, et al. (2017) Dermal substitute for reconstruction of large full thickness scalp defect: a case of dermal regeneration without removal of the outer table of the skull. ARC Journal of Surgery 3: 8-10.

8. Esser JFS (1918) Gestielte lokale nasenplastik mit zweizipfligen lappen: deckung des sekunderen defectes vom ersten zipfel durch den zweiten. Dtsch Z Chir 143: 385-390.

9. Zitelli JA (1989) The bilobed flap for nasal reconstruction. Arch Dermatol 125: 957-959.

10. Ibrahim AM, Rabie AN, Borud L, Tobias AM, Lee BT, et al. (2014) Common patterns of reconstruction for Mohs defects in the head and neck. J Craniofac Surg 25: 87-92.

11. Sutton AE, Quatela VC (1992) Bilobed flap reconstruction of the temporal forehead. Arch Otolaryngol Head Neck Surg 118: 978-982.

12. Tucker SB, Ransdell BL (2003) Delayed staged closure with repeated directional suturing of cutaneous defects. Dermatol Surg 29: 728734 